\title{
Incoherent interaction of nematicons in bias-free liquid-crystal cells
}

Yana Izdebskaya
yvi124@rsphysse.anu.edu.au

Vlad Shvedov

\section{Anton Desyatnikov}

\section{Wieslaw Krolikowski}

\section{Gaetano Assanto}

\section{Yuri Kivshar}

We study experimentally the propagation dynamics and interaction of a pair of mutually incoherent nematicons: spatial optical solitons in nematic liquid crystals. In contrast to earlier studies, we consider a bias-free liquid-crystal cell and compare the soliton interaction in copropagating and counterpropagating geometries. We analyze the dependence of nematicon interaction on input power and observe a direct manifestation of a long-range nonlocal nonlinearity. Attraction of counterpropagating solitons requires higher powers and longer relaxation times than that of copropagating nematicons due to losses-induced power asymmetry of counterpropagating nematicons. [DOI: 10.2971/jeos.2010.10008]

Keywords: nonlinear optics, liquid crystals, nematicons

\section{INTRODUCTION}

Solitons have been observed in diverse fields of nonlinear physics, and they share common fundamental properties originating from the interplay between nonlinear self-action of wave packets and their natural tendency to spread as they propagate. Spatial optical solitons [1], i.e. nonspreading selflocalized beams with the width unchanged during propagation, form due to a balance between linear diffraction and selffocusing in a nonlinear optical medium. These solitons have been investigated extensively in several nonlinear media, both in one- and two-dimensional geometries; they have significant potentials in signal processing, switching and readdressing in the future generation of all-optical circuits. In this context, a giant optical nonlinearity arising from molecular reorientation in nematic liquid crystals (NLCs) has attracted significant attention [2]-[4]. Both experimental [5, 6] and theoretical [7] results have been demonstrated for spatial optical solitons in nematics, also called nematicons [8].

Nematic liquid crystals consist of elongated molecules aligned along a given direction (known as the molecular director) owing to both anchoring at the boundaries and intermolecular forces [2]-[4]. The resultant medium exhibits a positive uniaxial anisotropy and birefringence, with ordinary and extraordinary refractive indices, $n_{\|}$and $n_{\perp}$, defined for polarizations parallel and orthogonal to the director. The reorientational nonlinearity allows generating nematicons at relatively low optical powers, in the $\mathrm{mW}$ region or below, for the study of the fundamental aspects and applications of light interaction with self-assembling nonlinear soft matter.

While initial studies of solitons in nematic liquid crystals considered the propagation of single nematicons [5, 9]-[14], the interaction of two copropagating nematicons of equal [15][19] or different wavelengths $[20,21]$ has been addressed more recently. Attraction and fusion of nematicons in bulk undoped NLCs in planar voltage-biased liquid crystal cell were reported by Peccianti et al. [15, 22].

Most of the studies considered nematicons propagating in the same direction. However, the interaction of counterpropagating solitons, e.g. in photorefractive crystals [23]-[26], is known to possess a rich physical behavior including convecments [31], and stabilization against them was achieved in a transversely periodic nonlinear medium [32]. In contrast to photorefractive solitons, there is little known about counterpropagating nematicons, although an experimental approach was developed based on the mutual deflection of two counterpropagating beams, which permitted to estimate the strength of thermal focusing in thick dye doped nematic-liquid-crystal samples excited by narrow laser beams $[33,34]$. In addition, recent numerical studies pointed out the possible occurrence of instabilities in counterpropagating solitary beams in liquid crystals $[35,36]$. tive dynamical instabilities [27]-[30], as observed in experi- 
In earlier experimental studies with nematicons the liquidcrystal cells were biased by an external electric field, aimed at controlling the orientation of the NLC molecules at rest and, therefore, their nonlinearity as well as their nonlocality [37], also affecting nematicon interactions. However, nematicons can exist in unbiased NLC cells provided the input beam is extraordinary polarized, e.g. with director and electric field coplanar with the plane $(x, z)$ parallel to the cell interfaces $[13,14,17,18,20,38]$. Because of the optical anisotropy of the liquid crystalline molecules and their birefringence, light beams propagating in NLC walk-off the direction of their wave vector. The walk-off can be adjusted by acting on the optic axis, i.e. by reorienting the molecular director [13, 39].

In this paper we experimentally address the propagation and interaction of copropagating (CO) and counterpropagating (CP) nematicons in a bias-free NLC cell. We investigate in detail both power- and time-dependent interactions of two identical solitons. In particular, we demonstrate that the longrange attraction of nonlocal solitons and their ability to form bound states are strongly affected by the geometry of the interaction.

\section{EXPERIMENTAL RESULTS}

We employ a cell in the geometry sketched in Figure 1(a), with two parallel polycarbonate plates separated by a $100 \mu \mathrm{m}$ gap. The cell contains the liquid crystal 6CHBT [40,41] which has negligible absorption and high nonlinearity with refractive indices $n_{e}=1.6718$ and $n_{o}=1.5225$ at room temperature. The polycarbonate plates are rubbed in the plane $(x, z)$ at the angle of $45^{\circ}$ with respect to the $z$ axis in order to maximize the alloptical change in refractive index for the extraordinary waves. Such prepared surfaces entail molecular orientation in a bulk analogous to that provided by pre-alignment via an external biasing field when the director is aligned along $z$. However, in contrast to the latter case, where the director can rotate in a plane orthogonal to the plates, here the optic axis remains parallel to them. The cell is sealed by two extra glass plates at opposite end-facets to avoid the formation of a meniscus, with rubbing along $x$, i.e. in the principal plane $x z$ of the extraordinary wave in bulk NLC, in order to prevent depolarization of the extraordinarily polarized input beam. The transition region from optic axis parallel to $x$ to the bulk alignment at $45^{\circ}$ with $z$ is adiabatic; hence, an $x$-polarized input beam remains an extraordinary eigenwave and can form a nematicon via reorientation $[13,14]$.

Figure 1(c) shows the experimental setup. Two identical beams are prepared using a system of mirrors and beam splitters. For CO solitons, two parallel input beams of extraordinary polarization (E-field along the $x$-axis) are formed using a standard Mach-Zehnder arrangement (beam splitters BS1 and BS2, mirrors M1 and M2). For CP solitons, the mirror M1 is removed and the beam transmitted by BS3 follows the route indicated by the dashed line. In addition, in the case of $\mathrm{CO}$ beams, the mirror M1 mounted on a piezoelectric transducer is made to vibrate (at a frequency of $1 \mathrm{kHz}$ ) to induce a Doppler shift on the reflected beam. Since the nonlinear response of NLC is slower than the mutual phase changes of the two beams $\sim 10^{-3} \mathrm{~s}$, such frequency detuning makes
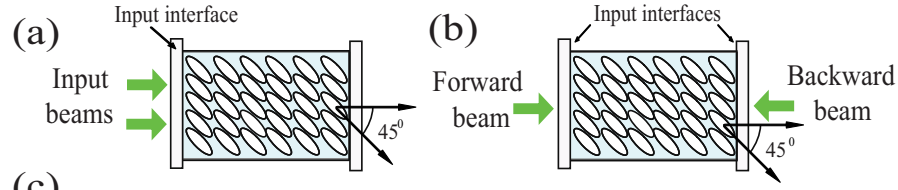

(c)

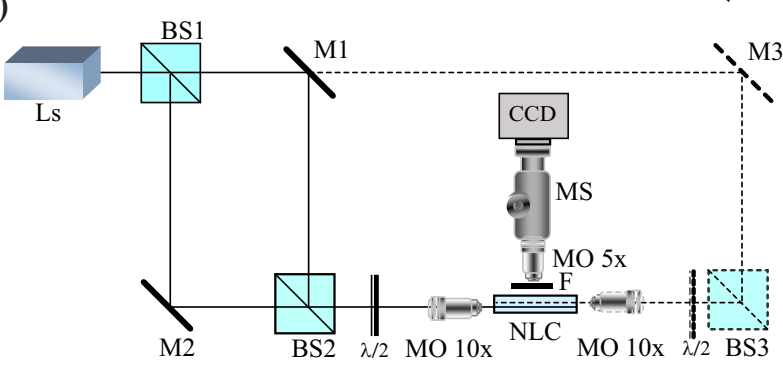

FIG. 1 Top view of the NLC planar cell for the study of (a) copropagating and (b) counterpropagating solitary beams. The ellipses indicate the orientation of the molecular director in the plane $(y, z)$. (c) Experimental setups for the copropagating (solid lines) and counterpropagating (dashed lines) cases: CW-laser ( $\mathrm{Ls}, \lambda=532 \mathrm{~nm}$ ), beam splitters $(B S)$, mirrors $(M)$, half wavelength plates $(\lambda / 2)$, microscope objectives $(M 0)$, cell $(\mathrm{NLC})$, filter (F), microscope (MS), and camera (CCD).

their interaction effectively incoherent. With the half-wave plate we control the polarization state of the two parallel Gaussian beams focused into the cell by a $10 \times$ objective. The waist of each beam is $w_{0}=2 \mu \mathrm{m}$ and the separation between them is $25 \mu \mathrm{m}$. The beam evolution inside the cell is monitored by collecting the light scattered above the cell with a $5 \times$ microscope-objective and a high resolution CCD camera. The total beam power in all experiments was kept low enough (below $15 \mathrm{~mW}$ ) to avoid thermo-optic effects due to absorption and heating.

First, we study the dynamics of the formation and interaction of two CO parallel beams in NLC as a function of the excitation power (see Figure 2). As discussed earlier [15], due to of the long-range character of the nonlocality, the nematicons attract each other progressively more for increasing input power. In most cases this attraction is independent of the relative phase of the solitons, even when they are mutually coherent. A sequence of images in Figure 2 shows the stationary trajectories of two initially parallel beams for different input powers. For low input power $(0.5 \mathrm{~mW})$, the self-focusing is too weak to overcome diffraction, so the beams keep spreading as they propagate. By increasing the power to $2 \mathrm{~mW}$, we achieve stable propagation of solitons and their weak attraction. Due to the high birefringence of the liquid crystal, both nematicons propagate at an angle with respect to the initial wave vector (directed along the $z$-axis). The measured walkoff of the Poynting vector is $\approx 4.3^{\circ}$. For higher excitation the attraction is so strong that it induces one (at $P \geq 3 \mathrm{~mW}$ ) or multiple $(P=13.5 \mathrm{~mW})$ intersections of the soliton trajectories. A quantitative analysis of this behavior is presented as a graph of nematicon distance $r$ versus propagation length $z$ for various input powers (see Figure 2(a)).

Next, we investigate the power-dependent dynamics of $\mathrm{CP}$ solitons in the same cell. In this case the beams are launched from opposite sides of the cell by focusing them with $10 \times$ micro-objectives (see Figures 1(a) and 1(c)). For better comparison with the $\mathrm{CO}$ case discussed above, we align the ini- 

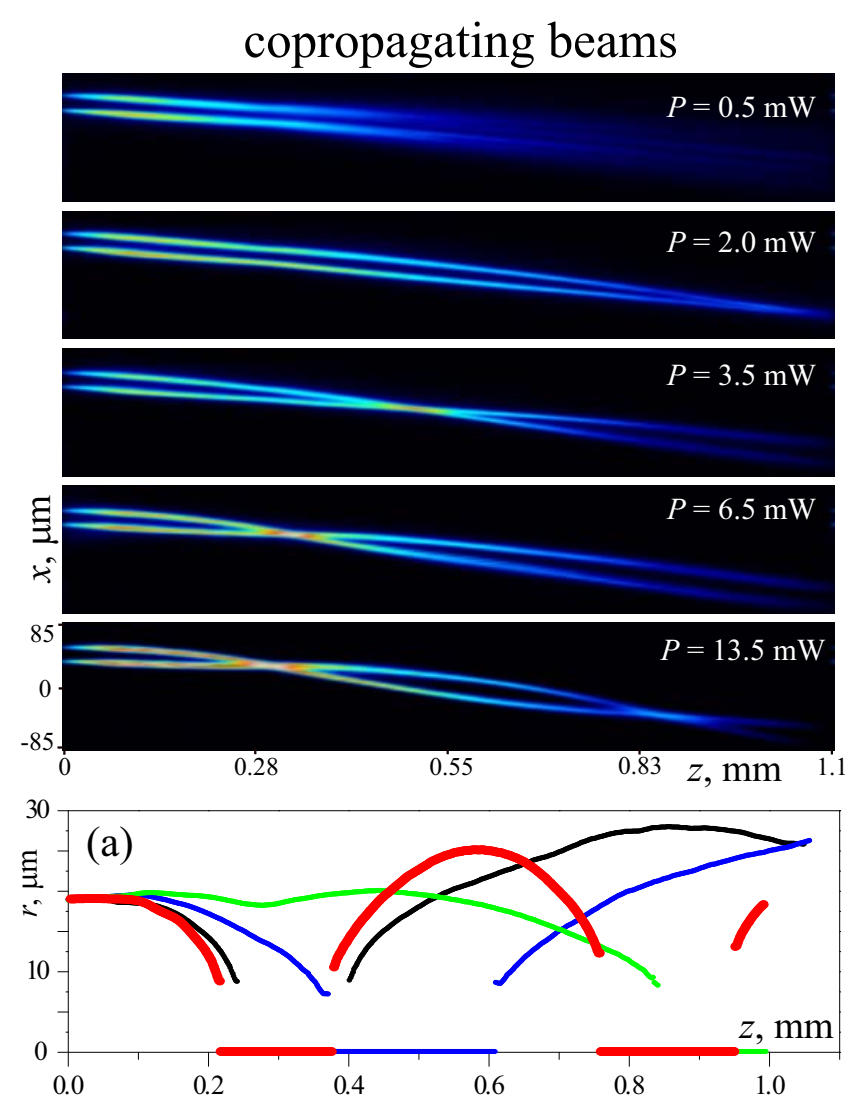

FIG. 2 Experimentally recorded images of light scattered from two CO nematicons at various input powers $P$ in each beam. (a) Measured distance $r$ between solitons versus propagation distance $z$ for various individual beam excitations: $2 \mathrm{~mW}$ (green line), $3.5 \mathrm{~mW}$ (blue line), $6.5 \mathrm{~mW}$ (black line), and $13.5 \mathrm{~mW}$ (red line).

tial trajectories (without walk-off and without interaction) to be parallel to the axis $z$ and spatially separated by $25 \mu \mathrm{m}$. As in the case of $\mathrm{CO}$ nematicons, the waist of each CP beam is $w_{0} \approx 2 \mu \mathrm{m}$.

Experimental results for $\mathrm{CP}$ beams are presented in Figure 3. As above, increasing the input power $(P \geq 2 \mathrm{~mW})$ leads to self-focusing and the formation of two CP nematicons. However, by further increasing the power we observe a clear difference in the trajectories of the $\mathrm{CP}$ nematicons as compared to the $\mathrm{CO}$ ones (compare Figures 2 and Figure 3). In particular, we find that the $\mathrm{CP}$ trajectories remain almost parallel up to a power of $5 \mathrm{~mW}$, while strong attraction in the $\mathrm{CO}$ case is already observed at about $2.5 \mathrm{~mW}$. For higher powers, the trajectories become closer to each other until they eventually merge into a single soliton at $13.5 \mathrm{~mW}$. In this regime the solitons propagate along the same trajectory forming a vector soliton with CP components. This behavior is illustrated in Figure 3(b) where we plot the separation between CP trajectories versus excitation. It is clear that the trajectories start approaching each other only after the input power exceeds $5 \mathrm{~mW}$. For higher powers, up to $18 \mathrm{~mW}$, we do not observe changes in soliton positions, neither associated with crossing, as in the $\mathrm{CO}$ case, nor due to of spatiotemporal instabilities. Figure 3(c) shows the intensity profiles for different input beam powers at the propagation distance $z=0.55 \mathrm{~mm}$.

In order to understand the origin of the different dynamics of $\mathrm{CO}$ and $\mathrm{CP}$ nematicons, we have to consider the role of
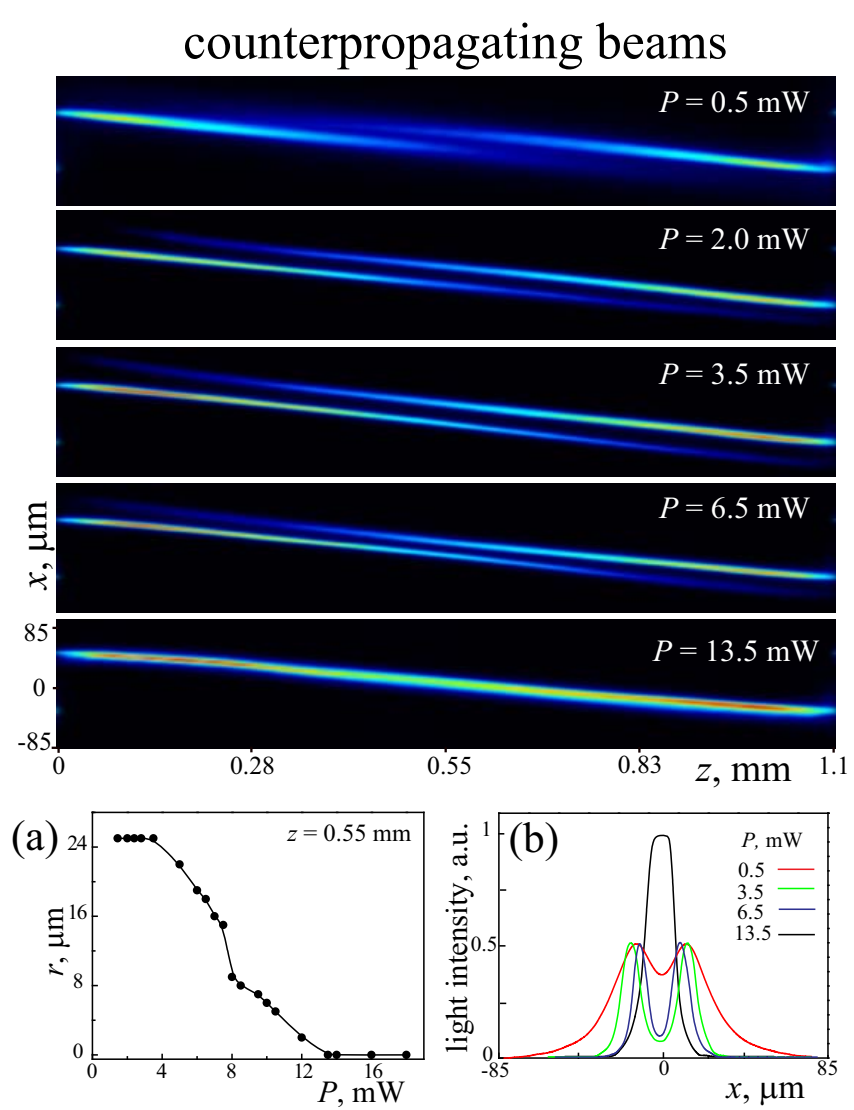

FIG. 3 Experimental results on the attraction between two CP solitons for various input powers. (a) Measured distance $r$ between solitons versus excitation, and (b) normalized intensity profiles for various input powers in each beam. Both (a) and (b) are acquired after a propagation distance $z=0.55 \mathrm{~mm}$.

scattering losses, which progressively reduce the beam power and the size of the nonlinear effect. While the two $\mathrm{CO}$ beams evolve forward with equal individual powers at each $z$, the $\mathrm{CP}$ beams interact with unequal intensities and sizes, i.e. with unbalanced strengths in the transverse force pulling the two nematicons towards each other. The two initially parallel CP solitons, say $\mathrm{A}$ and $\mathrm{B}$, launched at $z=0$ and $z=L$, respectively, tend to attract and shift the center of mass of the resulting dipole towards the more intense one, i.e. towards $\mathrm{A}$ for $z<L / 2$ and towards $\mathrm{B}$ for $z>L / 2$. This non-homogeneous force distribution results in a larger power requirement for the completion of the merging process as compared to the $\mathrm{CO}$ case.

The asymmetry between $\mathrm{CP}$ nematicons has a profound effect on the temporal dynamics of the interaction, with significant differences with the $\mathrm{CO}$ case. The acquired sequence of images from Video 1, displaying the temporal evolution of two initially parallel CO beams, which is presented in Figure 4 . These images clearly reflect the inertia of the nonlinear response. After light is switched on at $t=0 \mathrm{~s}$, the beams propagate linearly and diffract. It takes some time $(\sim 0.3 \mathrm{~s})$ for the solitons to form. Only after some additional time the soliton interaction becomes visible, leading to multiple intersections of their trajectories. In sharp contrast, the temporal evolution of $\mathrm{CP}$ beams is much slower as it requires $17 \mathrm{~s}$ for the solitons to merge and form a bound state as shown in Figure 4 and Video 2. 


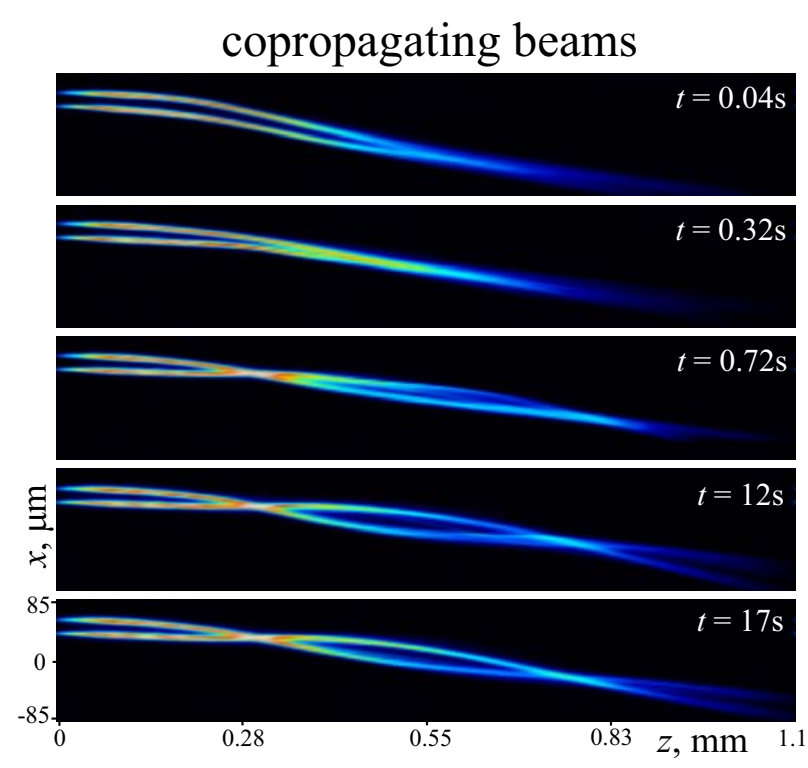

FIG. 4 Experimental results of the temporal evolution of two CO solitons shown in Figure 2 for power $13.5 \mathrm{~mW}$ (videosize: $550 \mathrm{kB}$, format: avi, see Fig4.avi).

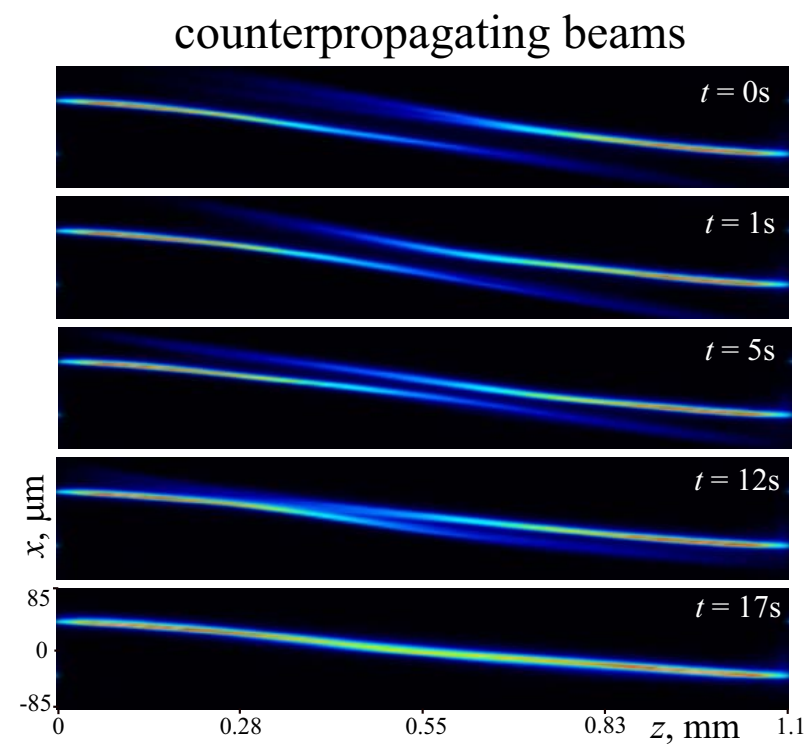

FIG. 5 Experimental results on the temporal evolution of the two CP nematicons shown in Figure 3 for power $13.5 \mathrm{~mW}$ (videosize: $350 \mathrm{kB}$, format: avi, see Fig5.avi).

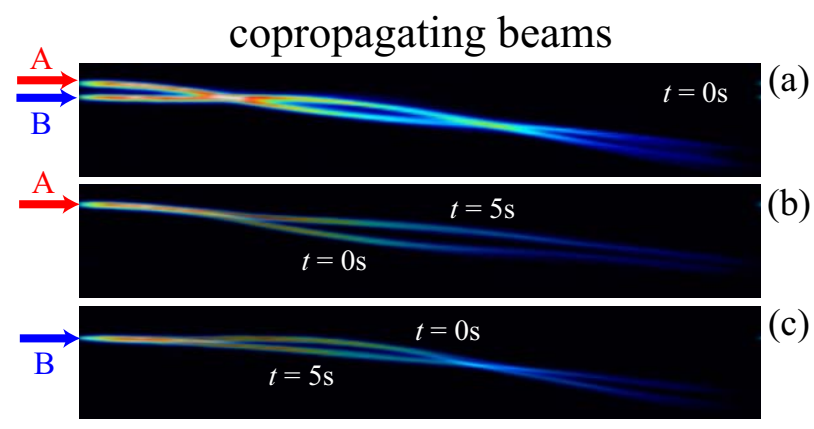

FIG. 6 (a) The bound CO nematicon pair at $t=0 \mathrm{~s}$ for the power of $13.5 \mathrm{~mW}$ and the initial separation of $25 \mu \mathrm{m}$ (the same as in bottom frame of Figure 2). (b) Superimposed intensity profiles of a single beam in the pair for $t=0 \mathrm{~s}$, and (c) for $t=5 \mathrm{~s}$ after the second beam has been blocked.

We stress here that the differences between $\mathrm{CO}$ and $\mathrm{CP}$ nematicons are solely due to their interaction as in our experi-

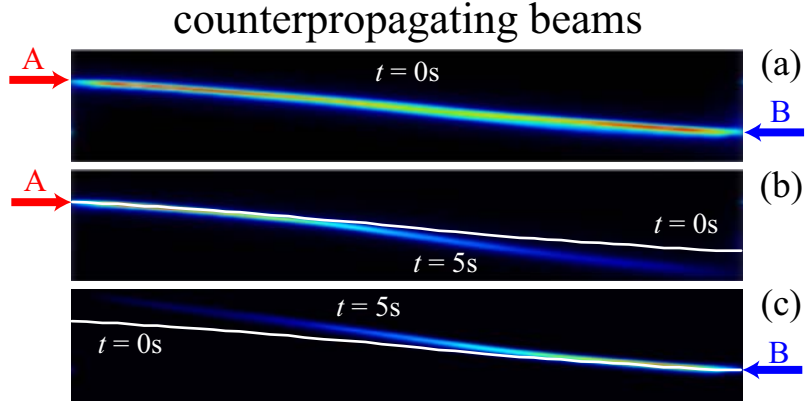

FIG. 7 The same as in Figure 6 for CP nematicons from the bottom frame of Figure 3. The white line in both (b) and (c) show the position on the intensity maximum of the coupled pair in (a).

ments all individual nematicons have identical characteristics. To underline this fact, we investigate the relaxation dynamics of each beam once its neighbor has been switched off at $t=0 \mathrm{~s}$. The results for $\mathrm{CO}$ and $\mathrm{CP}$ nematicons are presented in Figures 6 and 7 respectively. To highlight the differences, in Figures 6(b) and 6(c) we superimpose two images of the same beam, with $(t=0 \mathrm{~s})$ and without interaction $(t=5 \mathrm{~s})$, respectively. Similarly, the two "free" CP nematicons in Figures 7(b) and 7(c) are compared to their common (joint) waveguide (white line). In both cases the figures demonstrate how the remaining soliton reconstructs its original individual trajectory after about $5 \mathrm{~s}$. Importantly, this relaxation time is the same for both $\mathrm{CO}$ and CP cases because it is the time spent by a single nematicon to rid of the changes in director orientation induced by the interaction. Thus, the order-of-magnitude time difference necessary to build bound states in Figures 4 and 5 should be considered as the signature of symmetric $(\mathrm{CO})$ versus asymmetric (CP) interactions of nematicons.

\section{CONCLUSIONS}

We experimentally investigated generation, temporal dynamics and power-dependence of interacting copropagating and counterpropagating spatial solitons in unbiased nematic liquid crystals, outlining the major features in these two cases. In particular, we observed that the attraction of copropagating nematicons occurs at lower powers than for counterpropagating solitons, with an additional significant difference in time response between the two cases.

\section{ACKNOWLEDGEMENTS}

This work was supported by the Australian Research Council.

\section{References}

[1] Y. S. Kivshar, and G. P. Agrawal, Optical Solitons: From Fibers to Photonic Crystals (Academic Press, London, 2003).

[2] N. V. Tabirian, A. V. Sukhov, and B. Y. Zel'dovich, "Orientational optical nonlinearity of liquid crystals" Mol. Cryst. Liq. Cryst. 136, 1-139 (1986).

[3] I. C. Khoo, Liquid crystals: Physical Properties and Nonlinear Optical Phenomena (Wiley, New York, 1995).

[4] F. Simoni, Nonlinear Optical Properties of Liquid Crystals and Polymer Dispersed Liquid Crystals (World Scientific, Singapore, 1997). 
[5] M. Peccianti, G. Assanto, A. De. Luca, C. Umeton, and I. Khoo, "Electrically assisted self-confinement and waveguiding in planar nematic liquid crystall cells" Appl. Phys. Lett. 77, 7-9 (2000).

[6] X. Hutsebaut, C. Cambournac, M. Haelterman, J. Beeckman, and K. Neyts, "Measurement of the self-induced waveguide of a solitonlike optical beam in a nematic liquid crystal" J. Opt. Soc. Am. B 22, 1424-1431 (2005).

[7] D. W. McLaughlin, D. J. Muraki, and M. J. Shelley, "Self-focussed optical structures in a nematic liquid crystal" Physica D 97, 471497 (1996).

[8] G. Assanto, M. Peccianti, and C. Conti, “Nematicons: optical spatial solitons in nematic liquid crystals" Opt. Photonics News 14, 44-48 (2003).

[9] M. Peccianti and C. Assanto, "Signal readdressing by steering of spatial solitons in bulk nematic liquid crystals" Opt. Lett. 26, 16901692 (2001).

[10] C. Conti, M. Peccianti, and G. Assanto, "Route to nonlocality and observation of accessible solitons" Phys. Rev. Lett. 91, 073901 (2003).

[11] G. Assanto, and M. Peccianti, "Spatial solitons in nematic liquid crystals" IEEE J. Quantum Elect. 39, 13-21 (2003).

[12] C. Conti, M. Peccianti, and G. Assanto, "Observation of optical spatial solitons in a highly nonlocal medium" Phys. Rev. Lett. 92, 113902 (2004).

[13] M. Peccianti, C. Conti, G. Assanto, A. De Luca, and C. Umeton, "Highly anisotropic spatial solitons, modulational instability and their routing in liquid crystals" Nature 432, 733-737 (2004).

[14] A. Alberucci, M. Peccianti, G. Assanto, G. Coschignano, A. De Luca, and C. Umeton, "Self-healing generation of spatial solitons in liquid crystals" Opt. Lett. 30, 1381-1383 (2005).

[15] M. Peccianti, K. A. Brzdiakiewicz, and G. Assanto, "Nonlocal spatial soliton interactions in nematic liquid crystals" Opt. Lett. 27, 14601462 (2002).

[16] A. Fratalocchi, M. Peccianti, C. Conti, and G. Assanto, "Spiraling and cyclic dynamics of nematicons" Mol. Cryst. Liq. Cryst. 421, 197-207 (2004).

[17] A. Fratalocchi, A. Piccardi, M. Peccianti, and G. Assanto, "Nonlinearly controlled angular momentum of soliton clusters" Opt. Lett. 32, 1447-1449 (2007).

[18] A. Fratalocchi, A. Piccardi, M. Peccianti, and G. Assanto, "Nonlinear management of the angular momentum of soliton clusters: theory and experiments" Phys. Rev. A 75, 063835 (2007).

[19] C. García-Reimbert, A. A. Minzoni, T. R. Marchant, N. F. Smyth, and A. L. Worthy, "Dipole soliton formation in a nematic liquid crystal in the nonlocal limit" Physica D 237, 1088-1102 (2008).

[20] A. Alberucci, M. Peccianti, G. Assanto, A. Dyadyusha, and M. Kaczmarek, "Two-color vector solitons in nonlocal media" Phys. Rev. Lett. 97, 153903 (2006).

[21] G. Assanto, N. Smyth, and A. Worthy, "Two-color, nonlocal vector solitary waves with angular momentumn in nematic liquid crystals" Phys. Rev. A 78, 013832 (2008).

[22] M. Peccianti, C. Conti, G. Assanto, A. De Luca, and C. Umeton, "Alloptical switching and logic gating with spatial solitons in liquid crystals" Appl. Phys. Lett. 81, 3335-3337 (2002).

[23] 0. Cohen, R. Uzdin, T. Carmon, J. W. Fleischer, M. Segev, and S. Odoulov, "Collisions between optical spatial solitons propagating in opposite directions" Phys. Rev. Lett. 89, 133901 (2002).

[24] 0. Cohen, S. Lan, T. Carmon, J. A. Giordmaine, and M. Segev,
"Spatial vector solitons consisting of counterpropagating fields" Opt. Lett. 27, 2013-2015 (2002).

[25] M. Belic, P. Jander, A. Strinic, A. Desyatnikov, and C. Denz, "Selftrapped bidirectional waveguides in a saturable photorefractive medium" Phys. Rev. E 68, 025601 (2003).

[26] K. Motzek, M. Belic, T. Richter, C. Denz, A. Desyatnikov, P. Jander, and F. Kaiser, "Counterpropagating beams in biased photorefractive crystals: anisotropic theory" Phys. Rev. E 71, 016610 (2005).

[27] K. Motzek, P. Jander, A. Desyatnikov, M. Belic, C. Denz, and F. Kaiser, "Dynamic counterpropagating vector solitons in saturable self-focusing media" Phys. Rev. E 68, 066611 (2003).

[28] C. Rotschild, 0. Cohen, 0. Manela, T. Carmon, and M. Segev, "Interactions between spatial screening solitons propagating in opposite directions" J. Opt. Soc. Am. B 21, 1354-1357 (2004).

[29] M. Belic, P. Jander, K. Motzek, A. Desyatnikov, D. Jovic, A. Strinic, M. Petrovic, C. Denz, and F. Kaiser, "Counterpropagating selftrapped beams in photorefractive crystals" J. Opt. B-Quantum S. 0. 6, S190-S196 (2004).

[30] M. Belić, M. Petrović, D. Jović, A. Strinic, D. Arsenović, K. Motzek, F. Kaiser, Ph. Jander, C. Denz, M. Tlidi, and P. Mandel, "Transverse modulational instabilities of counterpropagating solitons in photorefractive crystals" Opt. Express 12, 708-716 (2004).

[31] M. Petrović, D. Jović, M. Belić, J. Schröder, Ph. Jander, and C. Denz, "Two dimensional counterpropagating spatial solitons in photorefractive crystals" Phys. Rev. Lett. 95, 053901 (2005).

[32] S. Koke, D. Träger, P. Jander, M. Chen, D. N. Neshev, W. Krolikowski, Y. S. Kivshar, and C. Denz, "Stabilization of counterpropagating solitons by photonic lattices" Opt. Express 15, 6279-6292 (2007).

[33] J. Henninot, M. Debailleul, and M. Warenghem, "Tunable nonlocality of termal nonlinearity in dye doped nematic liquied crystal" Mol. Cryst. Liq. Cryst. 375, 631-640 (2002).

[34] J. Henninot, J. Blach, and M. Warenghem, "Experimental study of the nonlocality of spatial optical solitons excited in nematic liquid crystal" J. Opt. A-Pure Appl. Op. 9, 20-25 (2007).

[35] A. Strinic, D. Jovic, M. Petrovic, D. Timotijevie, N. Aleksie, and M. Belic, "Counterpropagating beams in nematic liquid crystals" Opt. Express 14, 12310-12315 (2006).

[36] A. Strinic, D. Jovic, M. Petrovic, D. Timotijevie, N. Aleksie, and M. Belic, "Spatiotemporal instabilities of counterpropagating beams in nematic liquid crystals" 0pt. Mater. 30, 1213-1216 (2008).

[37] M. Peccianti, C. Conti, and G. Assanto, "Interplay between nonlocality and nonlinearity in nematic liquid crystals" Opt. Lett. 30, 415-417 (2005).

[38] A. Piccardi, G. Assanto, L. Lucchetti, and F. Simoni, "All-optical steering of soliton waveguides in dye-doped liquid crystals" Appl. Phys. Lett. 93, 171104 (2008).

[39] M. Peccianti, and G. Assanto, "Observation of power-dependent walk-off via modulational instability in nematic liquid crystal" 0pt. Lett. 30, 2290-2292 (2005).

[40] J. Baran, Z. Raszewski, R. Dabrowski, J. Kedzierski, and J. Rutkowska, "Some physical properties of mesogenic 4-(trans-4'n-Alkylcyclohexyl) isothiocyanatobenzenes" Mol. Cryst. Liq. Cryst. 123, 237-243 (1985).

[41] R. Dabrowski, J. Dziaduszek, and T. Szczucinski, "Mesomorphic characteristics of some new homologous series with the isothiocyanato terminal group" Mol. Cryst. Liq. Cryst. 124, 241-257 (1985). 